\title{
Mevalonate kinase deficiency and IBD: shared genetic background
}

Dear editor,

We read with interest the article entitled 'Monogenic diseases associated with intestinal inflammation: implications for the understanding of inflammatory bowel disease' written by Uhlig ${ }^{1}$ and published by Gut. The study, describing the very early onset of intestinal inflammation in several orphan monogenic diseases, aimed at determining the presence of a link between the IBD-like phenotype shown by these rare diseases and the intestinal inflammation seen in typical IBD. The IBD aetiology is multifactorial: at present, genome-wide association studies have identified 163 susceptibility loci associated with an increased risk of developing IBD. ${ }^{2}$ Beside these identified genetic loci that provide little contribution to explain IBD hereditability, the number of monogenic diseases presenting IBD-like symptoms is however continuously increasing. These monogenic diseases usually exhibit very early onset and very severe symptoms; in addition, they are often unresponsive to common drugs (anti-inflammatory and immunosuppressive treatments, such as anti-TNF $\alpha$ ).
Table 2 Genes and nucleotide variations (amino acid changes are reported in brackets, if present) found in homozygosis or heterozygosis in the six MKD patients analysed in our study

\begin{tabular}{|c|c|}
\hline Genes & Changes (nucleotide and amino acid) \\
\hline MUC4 NM_018406.6 (3q29) & $\begin{array}{l}\text { c. }{ }^{406 C>T ;} \text { c.G7702T (p.A2568S); c.A6447C (p.E2149D); c.A6409G } \\
\text { (p.T2137A); c.A6344T (p.D2115V); c.A6265G (p.I2089V); c.A6206G } \\
\text { (p.N2069S); c.C2834G (p.T945S) }\end{array}$ \\
\hline KRTAP4-8 NM_031960.2 (17q21.2) & $\begin{array}{l}\text { c. }{ }^{*} 468 \mathrm{~T}>\mathrm{C} ; c^{*}{ }^{*} 442 \mathrm{~T}>\mathrm{C} ; c^{*}{ }^{*} 258 \mathrm{~T}>A ; c .{ }^{*} 198 \mathrm{C}>A ; c .{ }^{*} 94 \mathrm{~T}>\mathrm{G} ; c^{*}{ }^{*} 30 \mathrm{~A}>\mathrm{G}, \\
\text { c.G502A (p.A168T); c.C451G (p.L151V); c.1dupA (p.M1fs) }\end{array}$ \\
\hline IL23R NM_144701.2 (1p31.3) & c.T929C (p.L310P) \\
\hline ADAM17 NM_003183.4 (2p25.1) & c. ${ }^{*} 290 A>G ; c .{ }^{*} 75 G>A ; c .{ }^{*} 61$ delA; c. $-172 T>C$ \\
\hline RET NM_020975.4 (10q11.2) & $c^{*}{ }^{*} 600 T>A ; c{ }^{*} 1506 G>A$ \\
\hline PTEN NM_001126049.1(10q23.3) & c. -1617 delA; c. $-1657 C>G$ \\
\hline IL10RA NM_001558.3 (11q23.3) & c.A1051G (p.R351G) \\
\hline GUCY2C NM_004963.3 (12p12.3) & c.T843G (p.F281L) \\
\hline PTPN2 NM_001207013.1(18p11.21) & c. ${ }^{*} 265 \mathrm{G}>\mathrm{A}$ \\
\hline HNF4A NM_000457.4 (20q13.12) & c. ${ }^{*} 906 \mathrm{~A}>\mathrm{C}$ \\
\hline
\end{tabular}

RefSeq mRNA (NM_.) and the chromosome localisation of each gene are reported.

MKD, mevalonate kinase deficiency.
In his article, ${ }^{1}$ Uhlig reports that children with very early onset of bowel inflammation may present a different phenotype and a different genetic architecture. In particular, these children can be classified either as classical IBD patients or as patients suffering from monogenic diseases, the latter also carrying a high number of genetic variants associated with susceptibility to develop IBD. In this regard, we wish to focus the attention on six patients suffering from mevalonate kinase deficiency (MKD, OMIM \#260920), diagnosed during the first year of life and followed-up at the Institute for Maternal and Child Health-IRCCS 'Burlo Garofolo' (Trieste, Italy). MKD, caused by inherited recessive mutations in the mevalonate kinase gene $(M V K)$, is characterised by febrile attacks, often associated with abdominal pain, diarrhoea and vomiting; it can be considered as an auto-inflammatory defect predisposing to IBD-like intestinal inflammation. ${ }^{3}{ }^{4}$ The six MKD patients were homozygous and/ or heterozygous for missense mutations on the MVK gene (table 1).
Abdominal pain arose in all MKD patients within the first year of life, with high values of both $\mathrm{C}$ reactive protein and erythrocyte sedimentation rate, and very frequent episodes of diarrhoea. Patients exhibit heterogeneous phenotypes: some are more severe and respond poorly to conventional drugs, while others are mild and respond quite well to treatments (anakinra, canakinumab, etanercept), despite carrying the same $M V K$ mutations.

We decided to further explore the hypothesis that MKD can be considered a monogenic cause of early onset pathologies with IBD-like symptoms. A whole exome sequencing analysing was carried out on the same six MKD patients followed-up at our children hospital, searching for genetic variants associated with chronic IBD. Exome enrichment was performed by TruSeq Exome Enrichment $62 \mathrm{Mb}$ (Illumina); sequence data were produced using Illumina HiSeq 1000 with 100-bp paired-end reads and analysed by CLC Genomics Workbench V.6.5 software.

The variants (table 2) were identified in genes known to be associated with IBD

Table 1 The table illustrates MVK gene mutations with RefSeq mRNA (NM_000431.2)

\begin{tabular}{|c|c|c|c|c|c|c|c|c|c|c|}
\hline Gene & $\mathrm{dbSNP}$ & Change & Ref & Obs & P1 & P2 & P3 & P4 & P5 & P6 \\
\hline MVK & rs104895334 & c.16_34del; p.Leu6_Gly12delinsGlyfs & CCTACTGGTGTCTGCTCCGG & $\mathrm{C}$ & WT & WT & WT & HET & WT & WT \\
\hline \multirow[t]{5}{*}{ NM_000431.2 (Chr 12) } & rs104895336 & c.G394A; p.V132I & $\mathrm{G}$ & A & HET & WT & WT & WT & WT & WT \\
\hline & rs104895297 & c.C404T; p.S135L & c & T & WT & WT & HET & WT & WT & WT \\
\hline & rs104895304 & c.T803C; p.1268T & $\mathrm{T}$ & C & WT & WT & WT & WT & HET & WT \\
\hline & rs104895358 & c.G1006A; p.G336S & G & A & WT & WT & WT & WT & WT & HOM \\
\hline & rs28934897 & c.G1129A; p.V377I & G & A & HOM & HOM & HET & HET & HET & WT \\
\hline
\end{tabular}

For each mutation, the following are shown: the respective identifier (dbSNP), the nucleotide substitution and, if present, the amino acid change (Change), the reference sequence (Ref) and the one observed (Obs). For each of the six patients (P1, P2, P3, P3, P4, P5, P6), the mutation is identified as wild-type (WT), heterozygous (HET) or homozygous (HOM). MVK, mevalonate kinase. 
from genome-wide association studies, and in genes responsible for monogenic intestinal diseases. Most of these genes encode for molecules of the innate or adaptive immunity (interleukin (IL)-23R, IL-10, IL-12B and STAT3) and for molecules which are fundamental for intestinal homeostasis (MUC4, GUCY2C, PTPN2, HNF4A and ADAM17). An extended supplementary table containing more detailed information about each patient's variants is available (https://drive.google. com/file/d/0B6i2Abl03rZLYXEyMDRjV GgtbEE/edit?usp=sharing.)

The different 'IBD-associated' variants, as identified in MKD patients with several combinations in both homozygous and heterozygous states, could account for the presence of abdominal symptoms and inflammation. Hence, we would like to reinforce the hypothesis of a shared genetic background between early onset IBD and MKD, which accounts for the common clinical and phenotypic features characterising the diseases.

Anna Monica Bianco, ${ }^{1}$ Martina Girardelli, ${ }^{1}$ Diego Vozzi, ${ }^{1}$ Sergio Crovella, ${ }^{1,2}$ Giulio Kleiner, ${ }^{1}$ Annalisa Marcuzzi ${ }^{1}$

${ }^{1}$ Health Genetics Unit, Institute for Maternal and Child Health—IRCCS "Burlo Garofolo", Trieste, Italy ${ }^{2}$ Medicine, Surgery and Health Sciences Department, University of Trieste, Trieste, Italy

Correspondence to Dr Annalisa Marcuzzi, Health Genetics Unit, Institute for Maternal and Child
Health—IRCCS "Burlo Garofolo", Via dell'Istria, Trieste 65/1-34137, Italy; annalisa.marcuzzi@burlo. trieste.it

Contributors AM, AMB and SC conceived and designed the study; $A M B$ and $M G$ analysed and interpreted the data and draw tables; DV and AMB performed whole genome sequencing bioinformatic analyses; SC, AM and GK redacted the manuscript; GK and $M G$ revised the manuscript for language and style. All authors have read and approved the final version of this manuscript.

Funding This study was supported by a grant from the Institute for Maternal and Child Health-IRCCS 'Burlo Garofolo'-Trieste, Italy (RC 42/2011).

Competing interests None.

Patient consent Obtained.

Ethics approval Independent Bioethics Committee of the IRCCS 'Burlo Garofolo'.

Provenance and peer review Not commissioned; internally peer reviewed.
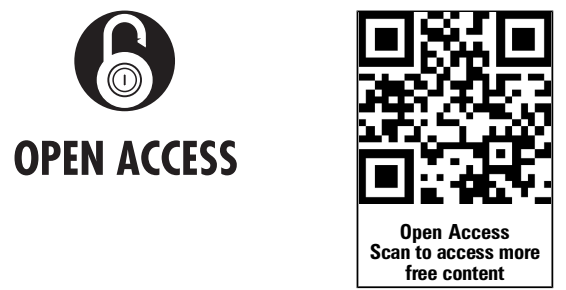

Open Access This is an Open Access article distributed in accordance with the Creative Commons Attribution Non Commercial (CC BY-NC 3.0) license, which permits others to distribute, remix, adapt, build upon this work non-commercially, and license their derivative works on different terms, provided the original work is properly cited and the use is noncommercial. See: http://creativecommons.org/licenses/ by-nc/3.0/

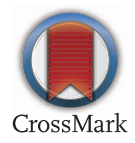

To cite Bianco AM, Girardelli M, Vozzi D, et al. Gut 2014:63:1367-1368.

Received 6 December 2013

Revised 27 December 2013

Accepted 30 December 2013

Published Online First 14 February 2014

Gut 2014;63:1367-1368

doi:10.1136/gutjn-2013-306555

\section{REFERENCES}

1 Uhlig HH. Monogenic diseases associated with intestinal inflammation: implications for the understanding of inflammatory bowel disease. Gut 2013;62:1795-805.

2 Franke A, McGovern DP, Barrett JC, et al. Genome-wide meta-analysis increases to 71 the number of confirmed Crohn's disease susceptibility loci. Nat Genet 2010;42:1118-25.

3 Patra KP, Dariya V, Thomas W, et al. Index of suspicion: case 1: leg cramps, hand spasms, diarrhea, and substantial weight loss in a 12 year old: case 2: hypothermia, hypoglycemia, and hyperbilirubinemia in a neonate: case 3: recurrent fevers, abdominal pain, and cervical lymphadenopathy in a 7 year old. Pediatr Rev 2011;32:299-305

4 Levy M, Arion A, Berrebi D, et al. Severe early-onset colitis revealing mevalonate kinase deficiency. Pediatrics 2013;132:e779-83. 